\title{
Generalized Topological Molecular Lattices
}

\author{
Kamal El-Saady ${ }^{1}$, Fatima Al-Nabbat ${ }^{2}$ \\ ${ }^{1}$ Department of Mathematics, Faculty of Science at Qena, South Valley University, Qena, Egypt \\ ${ }^{2}$ Department of Mathematics, College of Science, King Faisal University, Al-Hasa, Saudi Arabia \\ Email: el-saady@lycos.com, fatima math20@yahoo.com
}

Received 11 May 2015; accepted 7 July 2015; published 10 July 2015

Copyright (C) 2015 by authors and Scientific Research Publishing Inc.

This work is licensed under the Creative Commons Attribution International License (CC BY).

http://creativecommons.org/licenses/by/4.0/

(c) (7) Open Access

\begin{abstract}
The aim of this paper is to introduce the concept of generalized topological molecular lattices briefly GTMLs as a generalization of Wang's topological molecular lattices TMLs, Császár's setpoint generalized topological spaces and lattice valued generalized topological spaces. Some notions such as continuous GOHs, convergence theory and separation axioms are introduced. Moreover, the relations among them are investigated.
\end{abstract}

\section{Keywords}

Generalized Topological Molecular Lattices, Generalized Order Homomorphisms, Convergence of Molecular Nets, Separation Axioms

\section{Introduction}

In 1992 Wang [1], introduced his important theory called topological molecular lattice (briefly, TML) as a generalization of ordinary topological and fuzzy topological spaces in tools of molecules, remote neighborhoods and generalized order homomorphisms GOHs. Then many authors characterized some topological notions in such TMLs, such as convergence theories of molecular nets or ideals [1]-[3], separation axioms [1] [4] and other notions.

In this paper, we aim to introduce a generalization of TMLs under the name of generalized topological molecular lattice (briefly, GTML). In the same manner, we study several notions in these GTMLs, investigate some properties and set the relations among these notions including GOHs, convergence theories and separation axioms.

Throughout this work, $\left(L, \bigvee, \bigwedge,^{\prime}\right)$ is a complete lattice with an order-reversing involution ('), and with the smallest element $\perp$ and the largest element $\top \quad(\top \neq \perp)$.

By an $L$-generalized topology [5], on a non-empty ordinary set $X$, we mean a subfamily $\tau$ of $L^{X}$ with the following axioms: 
( $\mathrm{T}_{1}$ ) For all $\left\{A_{j}: j \in J\right\} \subseteq L^{X},\left\{A_{j}: j \in J\right\} \subseteq \tau \Rightarrow \bigvee_{j} A_{j} \in \tau$;

$\left(\mathrm{T}_{2}\right) \perp \in \tau$.

The pair $(X, \tau)$ is called an $L$-generalized topological space. Every element $A$ of $\tau$ is called $\tau$-open $L$-set and the pseudo complement $A^{\prime}$ is called $\tau$-closed $L$-set. The concept of $L$-generalized co-topological space can be defined dually.

For $A \in L^{X}$, the $L$-generalized interior of $A$ is the largest $L$-open subset contained in $A$ and denoted by $i(A)$, so $A$ is open if and only if $A=i(A)$. The $L$-generalized closure of $A$ is the smallest $L$-closed subset contains $A$ and denoted by $c(A)$, so $A$ is closed if and only if $A=c(A)$.

Let $f: X \rightarrow Y$ be an ordinary mapping. The corresponding $L$-fuzzy mapping $f^{\rightarrow}: L^{X} \rightarrow L^{Y}$ is defined as follows:

$$
f^{\rightarrow}(A)(y)=\bigvee\{A(x): x \in X, f(x)=y\}, \forall A \in L^{X}, \forall y \in Y
$$

and its inverse $f^{\leftarrow}: L^{Y} \rightarrow L^{X}$ defined as:

$$
f^{\leftarrow}(B)(x)=B(f(x)), \forall B \in L^{Y}, \forall x \in X
$$

A mapping $f^{\rightarrow}:(X, \tau) \rightarrow(Y, \mu)$ between two $L$-generalized topological spaces is said to be an $L$-generalized continuous mapping if and only if $\forall B \in \mu, f^{\leftarrow}(B) \in \tau$. The mapping $f^{\rightarrow}$ is called an $L$-generalized open (resp. $L$-generalized closed) mapping if $f^{\rightarrow}(G) \in \mu, \forall G \in \tau$ (resp. $f \rightarrow(F)$ is $\mu$-closed for all $\tau$-closed set $F$ ). The category of $L$-generalized topological spaces and their $L$-generalized continuous mappings is denoted by $\boldsymbol{L}$-GTop.

Let us recall that a non-zero element $a$ in a lattice $L$ is said to be a molecule, if for every $b, c \in L$ such that $a \leq b \vee c$, implies $a \leq b$ or $a \leq c$. Denote the set of all molecules of $L$ by $M(L)$ or $M$ for short, clearly, every element in $L$ can be constructed by elements of $M$, since each element in $L$ is a union of molecules.

Definition 1.1. [1] Let $L$ ba a complete lattice, $a \in L$. The subset $B \subseteq L$ is called a minimal family of a if the following two conditions are hold:

(i) $a=\bigvee B$.

(ii) If $D \subseteq L$ and $a \leq \bigvee D$, then $\forall b \in B, \exists d \in D$ such that $d \geq b$.

Denote the greatest minimal family of $a$ by $\beta(a)$. Hence, let $\beta^{*}(a)=\beta(a) \cap M(L)$.

It is easily to see that both $\beta(a)$ and $\beta^{*}(a)$ are minimal families of $a$.

Definition 1.2. [1] Let $L_{1}$ and $L_{2}$ be complete lattice. A mapping $f: L_{1} \rightarrow L_{2}$ is called a generalized order homomorphism or GOH for short if

(i) $f(a)=\perp$ if and only if $a=\perp$.

(ii) $f$ is join preserving, i.e.; $f\left(\bigvee_{j} a_{j}\right)=\bigvee_{j} f\left(a_{j}\right)$.

(iii) $f^{\vdash}$ is join preserving, where for all $b \in L_{2}, f^{\vdash}(b)=\left\{a \in L_{1}: f(a) \leq b\right\}$.

Theorem 1. [1] Let $f: L_{1} \rightarrow L_{2}$ be GOH, then the following properties are hold:

(1) $f$ and $f^{\vdash}$ are order preserving, i.e.:

$$
\begin{aligned}
& \forall a_{1}, a_{2} \in L_{1}, a_{1} \leq a_{2} \Rightarrow f\left(a_{1}\right) \leq f\left(a_{2}\right) . \\
& \forall b_{1}, b_{2} \in L_{2}, b_{1} \leq b_{2} \Rightarrow f^{\vdash}\left(b_{1}\right) \leq f^{\vdash}\left(b_{2}\right) .
\end{aligned}
$$

(2) $f^{\vdash} f(a) \geq a$, for all $a \in L_{1}$.

(3) $f f^{\vdash}(b) \leq b$, for all $b \in L_{2}$.

(4) $f(a) \leq b \Leftrightarrow a \leq f^{\vdash}(b)$.

(5) $f(a)=\bigwedge\left\{b \in L_{2}: f^{\vdash}(b) \geq a\right\}$, for all $a \in L_{1}$.

(6) $f^{\vdash}: L_{2} \rightarrow L_{1}$ is meet preserving, i.e.: $f^{\vdash}\left(\bigwedge_{j} b_{j}\right)=\bigwedge_{j} f^{\vdash}\left(b_{j}\right)$.

Theorem 2. [1] Let $f: L_{1}\left(M_{1}\right) \rightarrow L_{2}\left(M_{2}\right)$ be a GOH, then: 
(i) If $a \in M_{1}$, then $f(a) \in M_{2}$.

(ii) If $B$ is a minimal family of $a$ in $L_{1}$, then $f(B)$ is a minimal family of $f(a)$ in $L_{2}$.

Proposition 3. [1] Let $f: L_{1} \rightarrow L_{2}$, be a mapping between complete lattices. The following are equivalent:

(1) $f$ is an isomorphism.

(2) $f$ is a bijective $\mathrm{GOH}$.

(3) $f^{\vdash}$ is a bijective GOH.

\section{Main Notions in GTMLs}

This section is devoted to introduce the concept of generalized topological molecular lattices and other concepts which play an essential role in these GTMLs.

To denote a molecular lattice, the entry $L(M)$ is used: it indicates both the lattice itself and the set of its molecules.

Definition 2.1. Let $L(M)$ be a molecular lattice. A subfamily $\eta \subset L$ is said to be a generalized closed topology, or briefly, generalized co-topology, if

$\left(\mathrm{T}_{1}\right) \quad \eta$ is closed under arbitrary intersections;

$\left(\mathrm{T}_{2}\right) \quad T \in \eta$.

A generalized co-topology $\eta \subset L$ is said to be a closed topology (or co-topology) [1], if it satisfies the following additional conditions:

$\left(\mathrm{T}_{3}\right) \quad \eta$ is closed under finite union;

( $\left.\mathrm{T}_{4}\right) \perp \in \eta$.

The pair $(L(M), \eta)$ is called a generalized topological molecular lattice, or briefly, GTML.

Example 1. Let $(X, \mu)$ be a generalized topological space [6]. Then it is clear that $2^{X}$ is a molecular lattice and $\left(2^{X}, \eta\right)$ is a GTML, where $\eta=\{X \backslash G: G \in \mu\}$.

Example 2. Let $(X, \tau)$ be an L-generalized topological space [5]. Then we have that $\left(L^{X}, \eta\right)$ is a GTML, where $L^{X}$ is a molecular lattice and $\eta=\left\{A^{\prime}: A \in \tau\right\}$.

Definition 2.2. [7] Let $(L(M), \eta)$ be a GTML, $a \in M, F \in \eta$, and $a \not k F$. Then $F$ is said to be $a$ generalized remote neighborhood of $a$. The set of all generalized remote neighborhoods of a will be denoted by $\eta(a)$.

In a GTML, if $a \in M, F \in \eta(a)$ and $H \in \eta$ such that $H \leq F$, then we get $H \in \eta(a)$. However, for $F, H \in \eta(a), F \vee H$ need not to be in $\eta(a)$, since it does not necessary be closed element because $\eta$ is not necessary be closed under finite joins.

Definition 2.3. [8] Let $L$ be a complete lattice. A non empty subset $I$ of $L$ is said to be an ideal, if it satisfies the following conditions:

(i) For $a \in I, x \in L$ and $x \leq a \Rightarrow x \in I$.

(ii) For all $a, b \in I \Rightarrow a \vee b \in I$.

(iii) $\top \notin I$.

Generally, one can get that $\eta(a)$ is not necessary be an ideal in GTMLs. So, let us define the following:

$$
\eta^{*}(a)=\{F \in \eta(a): \forall H \in \eta(a), F \vee H \in \eta(a)\}
$$

Then $\eta^{*}(a) \subset \eta(a)$ is an ideal in GTMLs.

Definition 2.4. Let $(L(M), \eta)$ be a GTML and $A \in L$. The intersection of all $\eta$-elements containing $A$ will be called the generalized closure of A denoted by $A^{-}$. i.e.,

$$
A^{-}=\bigwedge\{F \in \eta: A \leq F\} .
$$

By the definition of $\eta$, one can obtain that $A$ is a closed element if and only if $A=A^{-}$.

Proposition 4 Let $(L(M), \eta)$ be a GTML, then the following statements are hold:

(1) $\forall A, B \in L$, if $A \leq B$, then $A^{-} \leq B^{-}$;

(2) $\forall A \in L, A \leq A^{-}$;

(3) $\forall A \in L,\left(A^{-}\right)^{-}=A^{-}$.

Proof.

(1) For $\forall A, B \in L$, with $A \leq B$, we have 


$$
B^{-}=\bigwedge\{F \in \eta: F \geq B\}=\bigwedge\{F \in \eta: F \geq B \geq A\} \geq \bigwedge\{F \in \eta: F \geq A\}=A^{-}
$$

i.e., $A^{-} \leq B^{-}$.

(2) For all $A \in L, A^{-} \geq A$. obvious;

(3) For all $A \in L$, we have $\left(A^{-}\right)^{-}=\bigwedge\left\{F \in \eta: F \geq A^{-} \geq A\right\}=A^{-}$.

Since any generalized co-topology is not necessarily closed under finite join, then the finite join is not necessarily be a closed $L$-fuzzy set, so some relations that are valid in topological molecular lattices do not remain true in generalized topological ones, for example the equation

$$
(A \vee B)^{-}=A^{-} \vee B^{-}
$$

is not necessarily true in generalized topological lattice as shown in the following example:

Example 3. Let $X=\{a, b, c\}$, and $\mu=\{\phi,\{a, c\},\{b, c\},\{b\}, X\}$ be a generalized topology on $X$. The class $\eta=\{X \backslash G: G \in \mu\}=\{X,\{b\},\{a\},\{a, c\}, \phi\}$ is a generalized co-topology on $2^{X}$. So for $A=\{a\}$ and $B=\{b\}$, we have that $A^{-}=\{a\}, B^{-}=\{b\}$ and $A^{-} \cup B^{-}=\{a, b\}$. But $A \cup B=\{a, b\}$ implies that $(A \cup B)^{-}=\{a, b\}^{-}=X$ which means that $(A \cup B)^{-} \neq A^{-} \cup B^{-}$.

Definition 2.5. Let $(L(M), \eta)$ be a GTML, $a \in M, A \in L$, then $a$ is said to be an adherence point of $A$, if for all $F \in \eta(a)$, we have $A \not F$.

Since $(L(M), \eta)$ is a GTML with $L$ equipped with an order reversing involution, we can define the generalized interior by

$$
A^{\circ}=\bigvee\left\{B: B \leq A, B^{\prime} \in \eta\right\}
$$

Proposition 5. Let $(L(M), \eta)$ be a GTML, then the following statements are hold:

(1) $\forall A \in L, A^{\circ} \leq A$.

(2) $\forall A, B \in L$, if $A \leq B$, then $A^{\circ} \leq B^{\circ}$

(3) $\forall A \in L,\left(A^{\circ}\right)^{\circ}=A^{\circ}$.

Proof.

(1) For all $A \in L, A^{\circ} \leq A$. obvious;

(2) For $\forall A, B \in L$, with $A \leq B$, we have

$$
A^{\circ}=\bigvee\left\{G: G \leq A, G^{\prime} \in \eta\right\}=\bigvee\left\{G: G \leq A \leq B, G^{\prime} \in \eta\right\} \leq \bigvee\left\{G: G \leq B, G^{\prime} \in \eta\right\}=B^{\circ}
$$

i.e., $A^{\circ} \leq B^{\circ}$.

(3) For all $A \in L,\left(A^{\circ}\right)^{\circ}=\bigvee\left\{G: G \leq A^{\circ} \leq A, G^{\prime} \in \eta\right\}=A^{\circ}$.

The relation $(A \cap B)^{\circ}=A^{\circ} \cap B^{\circ}$ which is true in TMLs, it is not true in GTMLs as shown in the following example:

Example 4. For $X=\{a, b, c\}$ and the generalized co-topology $\eta=\{X,\{b\},\{a\},\{a, c\}, \phi\}$ on $2^{X}$ as given in Example 3. Let $A=\{a, c\}$ and $B=\{b, c\}$, we have that $A^{\circ}=\{a, c\}, B^{\circ}=\{b, c\}$ and $A^{\circ} \cap B^{\circ}=\{c\}$. But $A \cap B=\{c\}$ implies that $(A \cap B)^{\circ}=\{c\}^{\circ}=\phi$ which means that $(A \cap B)^{\circ} \neq A^{\circ} \cap B^{\circ}$.

Definition 2.6. Let $\left(L_{1}\left(M_{1}\right), \eta_{1}\right)$ and $\left(L_{2}\left(M_{2}\right), \eta_{2}\right)$ be GTMLs. An GOH $f: L_{1} \rightarrow L_{2}$ is called:

(1) continuous GOH, if for every $H \in \eta_{2}$, we have $f^{\vdash}(H) \in \eta_{1}$.

(2) continuous at a molecule $a \in M_{1}$, if for every $H \in \eta_{2}(f(a))$, we have $\left(f^{\vdash}(H)\right)^{-} \in \eta_{1}(a)$.

It is clear that the generalized topological molecular lattices GTMLs and the continuous GOHs form a category denoted by GTML. 
Theorem 6. Let $\left(L_{1}\left(M_{1}\right), \eta_{1}\right)$ and $\left(L_{2}\left(M_{2}\right), \eta_{2}\right)$ be GTMLs, $f: L_{1} \rightarrow L_{2}$ be a GOH, then the following statements are equivalent:

(i) $f$ is a continuous GOH.

(ii) $\forall A \in L_{1}, f\left(A^{-}\right) \leq(f(A))^{-}$.

(iii) $\forall B \in L_{2},\left(f^{\vdash}(B)\right)^{-} \leq f^{\vdash}\left(B^{-}\right)$.

Proof. The proof the same as given for Theorem 5.2 [1].

For an $L$-generalized continuous mapping $f^{\rightarrow}: L^{X} \rightarrow L^{Y}$, it is well-known that $f^{\rightarrow}$ induced by an ordinary mapping $f: X \rightarrow Y$, and satisfied many useful properties ([9] [10]). Hence, the continuous GOHs can be regarded as a generalization of $L$-generalized continuous mappings.

Definition 2.7. Let $f:\left(L_{1}\left(M_{1}\right), \eta_{1}\right) \rightarrow\left(L_{2}\left(M_{2}\right), \eta_{2}\right)$ be an isomorphism and $f$ and $f^{-1}$ be continuous. A GOH $f: L_{1} \rightarrow L_{2}$ is said to be a homeomorphism.

Definition 2.8. Let $\left(L_{1}\left(M_{1}\right), \eta_{1}\right)$ and $\left(L_{2}\left(M_{2}\right), \eta_{2}\right)$ be GTMLs. A GOH $f: L_{1} \rightarrow L_{2}$ is said to be:

(1) closed, if for every $F \in \eta_{1}$, we have $f(F) \in \eta_{2}$

(2) open, if for every $A \in L_{1}\left(M_{1}\right)$ and every $B \in \eta_{1}$ such that $f^{\vdash}(A) \leq B$, there exists $H \in \eta_{2}$ such that $A \leq H$ and $f^{\vdash}(H) \leq B$.

Remark 1. In the case $L_{1}$ and $L_{2}$ are equipped with order reversing involutions, we can say that a GOH is open if it maps open elements in $\eta_{1}^{\prime}$ into open elements in $\eta_{2}^{\prime}$. Clearly, every L-generalized closed (resp. open) mapping is a closed GOH (resp. an open GOH).

As given in [1], we have the following easily established result.

Proposition 7 The compositions of closed (resp.,open) GOHs are closed (resp.,open) GOHs.

Definition 2.9. [1] Let $L(M)$ be a molecular lattice, $A \in L$ and $D$ is a directed set, then the mapping $S: D \rightarrow M$ is called a molecular net and denoted by $S=\{S(n): n \in D\}$. S is said to be in $A$, if $S(n) \leq A, \forall n \in D$.

Definition 2.10. [1] Let $L(M)$ be a molecular lattice, $S=\{S(n): n \in D\}$ and $T=\{T(m): m \in E\}$ be two molecular nets, then $T$ is said to be a subnet of $S$, if there exists a mapping $g: E \rightarrow D$ such that

(i) $T=S \circ g$.

(ii) $\forall n \in D, \exists m \in E$ such that $g(k) \geq n, \forall m \leq k \in E$.

Definition 2.11. Let $(L(M), \eta)$ be a GTML, $S=\{S(n): n \in D\}$ be a molecular net and $a \in M$, then:

(1) $a$ is called a limit point of $S$, if $\forall F \in \eta(a), S(n) \not F$ eventually true, and denoted by $S \rightarrow a$. The join of all limit points of $S$ will be denoted by $\lim S$.

In symbol, $\lim S=\bigvee\{x \in M: S \rightarrow x\}$.

(2) $a$ is called a cluster point of $S$, if $\forall F \in \eta(a), S(n) \npreceq F$ frequently true, and denoted by $S \infty a$. The join of all cluster points of $S$ will be denoted by cluS .

In symbol, $c l u S=\bigvee\{x \in M: S \infty x\}$.

Corollary 1 [1] Suppose that $S \rightarrow a$ (resp., $S \infty a$ ) and $b \leq a$. Then $S \rightarrow b$ (resp., $S \infty b$ ).

From the Definition 1.1, similarly to the case of TMLs, the following proposition is hold:

Proposition 8. Let $(L(M), \eta)$ be a GTML, $S: D \rightarrow M$ be a molecular net and $a \in M$, then:

(1) $S \rightarrow a$ if and only if $\forall b \in \beta^{*}(a), S \rightarrow b$.

(2) $S \propto a$ if and only if $\forall b \in \beta^{*}(a), S \infty b$.

Proposition 9. Let $(L(M), \eta)$ be a GTML, $S: D \rightarrow M$ be a molecular net and $a \in M$, then:

(1) $S \rightarrow a$ if and only if $a \leq \lim S$.

(2) $S \propto a$ if and only if $a \leq c l u S$. 
Proof.

(1) We only prove the sufficiency. Suppose that $a \leq \lim S$ and $\beta^{*}(a)$ is a standard minimal family of $a$. Since $\lim S=\bigvee\{x \in M: S \rightarrow x\} \geq a$. Then for all $y \in \beta^{*}(a)$, there exists a limit point $x$ of $S$ such that $y \leq x$. By Corollary 1, $S \rightarrow y$ and therefore $S \rightarrow a$.

(2) The proof is similar to that of (1) and is omitted.

Remark 2. Let $L(M)$ be a molecular lattice, $S$ be a molecular net and $a \in M$, then

(1) If $S=\{S(n): n \in D\}$ is a constant net, i.e., $\forall n \in D, S(n)=a$, then $S \rightarrow a$.

(2) If $S \rightarrow a$ and $T$ be a subnet of $S$, then $T \rightarrow a$.

Theorem 10 [8] Let $(L(M), \eta)$ be $a G T M L, A \in L$ and $a \in M$, then:

(1) If $a \leq A^{-}$, then there exists a molecular net $S: D \rightarrow M$ in $A$, such that $S \rightarrow a$.

(2) If $S: D \rightarrow M$ is a molecular net in $A$, such that $S \infty a$, then $a \leq A^{-}$.

Corollary 2 [8] Let $(L(M), \eta)$ be a GTML, $A \in L$ and $a \in M$, then $a \leq A^{-}$if and only if there exists a molecular net $S: D \rightarrow M$ in A, such that $S \rightarrow a$.

\section{Separation Axioms in GTMLs}

In this section, we introduce some kinds of separation axioms in GTMLs and investigate their properties. Moreover, we discuss the relations among them, isomorphic GOHs.

Definition 3.1. Let $(L(M), \eta)$ be a GTML, then

(1) $(L(M), \eta)$ is called $G T_{-1}$, if $\forall a, b \in M, a<b$, there exists $F \in \eta^{*}(b)$ such that $a \leq F$.

(2) $(L(M), \eta)$ is called $G T_{0}$, if $\forall a, b \in M, a \neq b$, there exists $F \in \eta^{*}(b)$ such that $a \leq F$ or there exists $H \in \eta^{*}(a)$ such that $b \leq H$.

(3) $(L(M), \eta)$ is called $G T_{1}$, if $\forall a, b \in M, a \leq b$, there exists $H \in \eta^{*}(a)$ such that $b \leq H$.

(4) $(L(M), \eta)$ is called $G T_{2}$, if $\forall a, b \in M, a \wedge b=\perp$, there exists $H \in \eta^{*}(a)$ and $F \in \eta^{*}(b)$ such that $H \vee F=\top$.

According to the above definitions, we can directly obtain the following results:

Corollary 3 For a GTML, we have the following implications:

$$
\begin{gathered}
G T_{1} \Rightarrow G T_{0} \Rightarrow G T_{-1} . \\
G T_{2}+G T_{-1} \Rightarrow G T_{1} .
\end{gathered}
$$

In general, we have that $G T_{2}$ does not imply $G T_{1}$. The next example [1] is clear.

Example 5. Take $L=[0,1]$ and $\eta=\{0,1\}$, then clearly that $(L(M), \eta)$ is not $G T_{-1}$ and hence it is not $G T_{1}$. But there are no disjoint points, so $(L(M), \eta)$ is $G T_{2}$.

Definition 3.2. [1] Let $L(M)$ be a molecular lattice, $A \in L$ with $A \neq \perp, m \in M$, then $m$ is called a component of $A$, if $m \leq A$ and $m^{\prime} \in M$ with $m^{\prime} \geq m$ and $m^{\prime} \leq A$ imply that $m^{\prime}=m$.

Lemma 1. [1] Let $L(M)$ be a molecular lattice, $A \in L$ with $A \neq \perp, a<A$ and $m \in M$, then $A$ has at least one component $m$ such that $a \leq m$.

Theorem 11. Let $(L(M), \eta)$ be a GTML, then it is $G T_{-1}$, if and only if $\forall a \in M$, we have $a$ is $a$ component of $a^{-}$.

Proof.

Assume that there exists $a \in M$ such that $a$ is not a component of $a^{-}$, then by the preceding lemma, we can choose a component $b$ of $a^{-}$such that $a<b$. Since $(L(M), \eta)$ is a $G T_{-1}$, then $\exists F \in \eta^{*}(b)$ such that $a \leq F$. Hence $a^{-} \leq F$ and so $b \leq F$. a contradiction.

Let $a, b \in M$ with $a<b$, then by the assumption, we have $a$ is a component of $a^{-}$and hence $b \leq a^{-}$. Then $a^{-} \in \eta^{*}(b)$ and $a \leq a^{-}$.

Therefore, $(L(M), \eta)$ is $G T_{-1}$. 
Theorem 12. Let $(L(M), \eta)$ be a GTML, then it is $G T_{0}$, if and only if $\forall a, b \in M, a \neq b$, we have $a \nless b^{-}$ or $b \leq a^{-}$.

Proof.

Let $(L(M), \eta)$ be $G T_{0}$, then $\forall a, b \in M, a \neq b$, there exists $F \in \eta^{*}(b)$ such that $a \leq F$ or there exists $H \in \eta^{*}(a)$ such that $b \leq H$. Hence, $a$ is not an adherence point of $b$ or $b$ is not an adherence point of $a$ which implies that $a \leq b^{-}$or $b \pm a^{-}$.

Let $a, b \in M$ with $a \neq b$, then we have $a \not b^{-}$or $b \not a^{-}$. Hence, we get $b^{-} \in \eta^{*}(a)$ with $b \leq b^{-}$or $a^{-} \in \eta^{*}(b)$ with $a \leq a^{-}$which complete the proof.

Theorem 13. Let $(L(M), \eta)$ be a GTML, then it is $G T_{1}$, if and only if $\forall a \in M$, a is closed.

Proof.

Let $(L(M), \eta)$ be $G T_{1}$ and $a, b \in M$, if $a \not \leq b$, then there exists $H \in \eta^{*}(a)$ such that $b \leq H$. Hence, $a$ is not an adherence point of $b$. Thus, $b$ contains all its adherence points and hence, $b$ is closed.

Let $a, b \in M, a \leq b$. By the assumption, $b$ is closed and then $b \in \eta^{*}(a)$ with $b \leq b$. Therefore, $(L(M), \eta)$ is $G T_{1}$.

Theorem 14. Let $(L(M), \eta)$ be a GTML, then it is $G T_{2}$, if and only if for every molecular net $S$, limS contains no disjoint molecules.

Proof.

Let $S=\{S(n): n \in D\}$ be a molecular net such that $a, b \leq \operatorname{limS}$ with $a \wedge b=\perp$. Let $F \in \eta^{*}(a)$ and $H \in \eta^{*}(b)$, since $S \rightarrow a$, then $S \nless F$ eventually, i.e.; $\exists n_{0} \in D$ such that $\forall n \in D, n \geq n_{0}$ we have $S(n) \nless F$. Similarly, we have $S(n) \npreceq H$, but $D$ is a directed set, hence $\exists k \in D$ such that $S(k) \nsubseteq F$ and $S(k) \nless H$. Then $S(k) \nless F \vee H$. Therefore, $F \vee H \neq \top$, then $(L(M), \eta)$ is not $G T_{2}$.

Assume that $(L(M), \eta)$ is not $G T_{2}$, then $\exists a, b \in M$ with $a \wedge b=\perp$ and $\forall F \in \eta^{*}(a), H \in \eta^{*}(b)$, we have $F \vee H \neq \top$. Thus, we can choose a molecule $S((F, H))$ such that $S((F, H)) \neq F \vee H$. Put

$$
S=\left\{S((F, H)):(F, H) \in \eta^{*}(a) \times \eta^{*}(b)\right\}
$$

Then $S$ is a molecular net with both $a$ and $b$ are limit points of $S$. Hence, limS contains at least two disjoint molecules.

Theorem 15. Let $\left(L_{1}\left(M_{1}\right), \eta_{1}\right)$ and $\left(L_{2}\left(M_{2}\right), \eta_{2}\right)$ be GTMLs. If $\left(L_{1}\left(M_{1}\right), \eta_{1}\right)$ is a $G T_{i}(i=-1,0,1,2)$ and $f: L_{1} \rightarrow L_{2}$ be a homeomorphism, then so is $\left(L_{2}\left(M_{2}\right), \eta_{2}\right)$.

Proof. We only show the case of $G T_{2}$ and the others are similar. Let $\left(L_{1}\left(M_{1}\right), \eta_{1}\right)$ be a $G T_{2}$ and $x, y \in M_{2}$ with $x \wedge y=\perp$. Since $f$ is bijective, then there exist $a, b \in M_{1}$ such that $f(a)=x$ and $f(b)=y$. Then there exist $H \in \eta_{1}^{*}(a)$ and $F \in \eta_{1}^{*}(b)$ such that $H \vee F=\top$. But $f$ is isomorphic GOH, so $f(H) \in \eta_{2}^{*}(x)$ and $f(F) \in \eta_{2}^{*}(y)$.

Thus, $f(H) \vee f(F)=f(H \vee F)=f(\top)=\top$.

Therefore, $\left(L_{2}\left(M_{2}\right), \eta_{2}\right)$ is also a $G T_{2}$.

Similarly, one can check the other cases.

Analogously to [4], we give the next definitions:

Definition 3.3. Let $(L(M), \eta)$ be a GTML, then $(L(M), \eta)$ is said to be $G U_{2}$, if and only if $\forall a, b \in M$ 
with $a \wedge b=\perp$, there exists $H \in \eta^{*}(a)$ and $F \in \eta^{*}(b)$ such that $H^{\circ} \vee F^{\circ}=\top$.

Clearly, if $(L(M), \eta)$ is a $G U_{2}$, then it is $G T_{2}$. Furthermore, we have the following:

Definition 3.4. Let $(L(M), \eta)$ be a GTML, then $(L(M), \eta)$ is called an interior additive if $\forall F, H \in \eta$, we have $H^{\circ} \vee F^{\circ}=(H \vee F)^{\circ}$.

Theorem 16. Let $(L(M), \eta)$ be a GTML, if it is both $G T_{2}$ and an interior additive, then $(L(M), \eta)$ is a $G U_{2}$.

Proof. Let $a, b \in M$ with $a \wedge b=\perp$. Since $(L(M), \eta)$ is $G T_{2}$, then there exists $H \in \eta^{*}(a)$ and $F \in \eta^{*}(b)$ such that $H \vee F=\top$. Hence, $(H \vee F)^{\circ}=\top$.

But $(L(M), \eta)$ is an interior additive, then $H^{\circ} \vee F^{\circ}=(H \vee F)^{\circ}=(\top)^{\circ}=\top$.

Therefore, $(L(M), \eta)$ is a $G U_{2}$.

\section{Conclusion}

The concept of generalized topological molecular lattices GTMLs has been defined. Some notions have been extended to such spaces namely continuous GOHs, convergence theory in terms of molecular nets and and separation axioms.

\section{References}

[1] Wang, G.J. (1992) Theory of Topological Molecular Lattices. Fuzzy Sets and Systems, 47, 351-376. http://dx.doi.org/10.1016/0165-0114(92)90301-J

[2] Chen, Y. (1996) Convergence in Topological Molecular Lattices. Fuzzy Sets and Systems, 84, 97-102. http://dx.doi.org/10.1016/0165-0114(95)00224-3

[3] Bai, S.Z. (1997) Q-Convergence of Ideals in Fuzzy Lattices and Its Applications. Fuzzy Sets and Systems, 92, 357-363. http://dx.doi.org/10.1016/S0165-0114(96)00174-1

[4] Chen, S.L. and Wu, Z.X. (2001) Urysohn Separation Property in Topological Molecular Lattices. Fuzzy Sets and Systems, 123, 177-184. http://dx.doi.org/10.1016/S0165-0114(00)00115-9

[5] El-Saady, K. and Al-Nabbat, F. On Lattice-Valued Generalized Topological Spaces. Submittted for Publication.

[6] Csàszàr, À. (2002) Generalized Topology, Generalized Continuity. Acta Mathematica Hungarica, 96, 351-357. http://dx.doi.org/10.1023/A:1019713018007

[7] Al-Nabbat, F. (2015) Lattice-Valued Generalized Neighborhood Structures. To Appear in Southeast Asian Bulletin of Mathematics.

[8] Yang, Z.Q. (1986) Ideals in Topological Molecular Lattices. Acta Mathematica Sinica, 29, 276-279.

[9] Chang, C.L. (1968) Fuzzy Topological Spaces. Journal of Mathematical Analysis and Applications, 24, 182-190. http://dx.doi.org/10.1016/0022-247X(68)90057-7

[10] Liu, Y.M. and Luo, M.K. (1997) Fuzzy Topology. World Scientific, Singapore. 